\section{People-plant Relationships in an Office Workplace: Perceived Benefits for the Workplace and Employees}

\author{
Jane Dyrhauge Thomsen ${ }^{1}$ \\ Department of Agriculture and Ecology, Faculty of Life Science, University \\ of Copenhagen, Højbakkegaard Allé 13, DK-2630 Taastrup, Denmark
}

Hans K.H. Sønderstrup-Andersen

Department of Psychology and Educational Studies, Roskilde University, Universitetsvej 1, DK-4000 Roskilde, Denmark

\section{Renate Müller \\ Department of Agriculture and Ecology, Faculty of Life Science, University of Copenhagen, Højbakkegaard Allé 13, DK-2630 Taastrup, Denmark}

Additional index words. human issues in horticulture, indoor plants, environmental psychology, qualitative research, occupational health

\begin{abstract}
The study presented in this article represents an initial attempt to generate indepth information about how ornamental plants in real-life office workplaces interact with workplace characteristics, thus influencing working environment and well-being of the employees. Using a qualitative, explorative, and inductive case-study design, the study provides an example of how a cross-disciplinary unit engaged in administrative office work at a Danish institution applied ornamental plants. The results document that ornamental plants are an integrated part of the workplace. The employees used ornamental plants in numerous ways to either actively manipulate different aspects of the surroundings or more passively cope with demands from the surroundings. Furthermore, the use of the ornamental plants was structured by a number of factors: culture and traditions, provisional orders, organizational structures, practices, values and history, company policies, and characteristics of the indoor architectural environment. Ornamental plants were perceived as affecting many aspects of the working environment (e.g., the physical surroundings, the social climate, image of the workplace, etc.), the individual's well-being (e.g., mood, general well-being, emotions, self confidence, etc.), and to some degree the workplace's competitiveness. However, the actual effects were the results of a complex interaction among the way the ornamental plants were applied, characteristics of the present ornamental plants (e.g., size, species and condition), and characteristics of the individual employee (e.g., personal experiences, preferences, and values).
\end{abstract}

Researching the impact of various elements of the working environment is important to secure the company's competitiveness as well as the well-being of the employees. Ornamental plants are one element we know relatively little about. However, they are often an integrated part of the interior in office settings and have been investigated in various studies (Conklin, 1978; Pearson-Mims and Lohr, 2000; Sundstrom and Sundstrom, 1986).

A number of studies investigating peopleplant relationships in office settings have been carried out during the last two decades (Adachi et al., 2000; Bringslimark et al., 2007; Campbell, 1979; Chang and Chen, 2005;

Received for publication 15 Oct. 2010. Accepted for publication 31 Mar. 2011.

We thank the participants in this study for delivering valuable inputs. Furthermore, we thank Eva Rosenqvist, Libere Nukurunziza, and Henrik Vlk Lütken for helping with analyzing the data.

${ }^{1}$ To whom reprint requests should be addressed; e-mail jdt@life.ku.dk. is influenced by the density of plants (Larsen et al., 1998) and whether plants can be seen from the workstation (Bringslimark et al., 2007). Other studies point out that the task type is of significant importance (Shibata and Suzuki, 2001, 2002, 2004). Specifically, ornamental plants seem to have a positive effect on creative task performance but a negative impact on routine task performance (Shibata and Suzuki, 2002). However, presumably over time, ornamental plants might actually lead to increased routine task performance, because their presence supports recovery from mental fatigue (Shibata and Suzuki, 2001).

\section{Stress and Discomfort Symptoms}

Along this line, some studies have investigated office plants' ability to reduce stress symptoms (Bringslimark et al., 2007; Chang and Chen, 2005; Lohr et al., 1996). It is difficult to draw a coherent conclusion from the research as a result of differences between the studies in terms of how the concept of stress is defined and used. Therefore, it can be questioned whether the studies are investigating the same phenomenon, which is further complicated by the fact that there is no consensus in the field on how to define workrelated stress (Sonnentag and Frese, 2003). However, it is obvious that the presence of plants in office settings can reduce physical discomfort symptoms (Fjeld, 2000; Fjeld et al., 1998). Nevertheless, a small but significant reliable association with higher sick leave has been found (Bringslimark et al., 2007).

\section{Mood and Emotions}

It has been found that ornamental plants in workplaces can improve mood and invoke positive feelings (Adachi et al., 2000; Shibata and Suzuki, 2002, 2004). Nevertheless, it has also been indicated that gender (Shibata and Suzuki, 2002, 2004), whether the plants are flowering or not (Adachi et al., 2000), and task type (Shibata and Suzuki, 2001, 2002, 2004) are all aspects that play an moderating role. and Suzuki, 2001, 2002, 2004; Shoemaker et al., 1992). All the studies are characterized by using experimental or quasi-experimental research designs to test the effect of ornamental plants on a wide range of factors such as productivity, stress, and discomfort symptoms; mood and emotions; job satisfaction; and attitudes toward the workplace, as reviewed in the following four paragraphs.

\section{Productivity}

One of the most investigated aspects is how ornamental plants influence productivity (Bringslimark et al., 2007; Larsen et al., 1998; Lohr et al., 1996; Shibata and Suzuki, 2001, 2002, 2004). The results of these studies indicate that the presence of ornamental plants seems to foster increased productivity. However, it has also been suggested that the effect is moderated by other factors. For example, it has been found that productivity

\section{Job Satisfaction and Attitudes toward the Workplace}

Job satisfaction and attitudes toward the workplace have also been investigated (Dravigne et al., 2008; Shoemaker et al., 1992). The studies obtained contradictory results. Dravigne et al. (2008) found that ornamental plants and window views of nature were positively associated with higher job satisfaction. However, they did not report whether the effect was caused by the window views or the plants. On the other hand, Shoemaker et al. (1992) found no significant effects. However, the Shoemaker et al. (1992) survey study had a low return rate, which might add to the fact that the results of the two studies are contradictory.

Beneficial effects on the working environment in office settings and thereby the well-being of the employees have been 
indicated. However, the results seem ambiguous. The ambiguity might be caused by heterogeneity in experimental manipulations, settings, samples, exposure durations, measurements, and the lack of a coherent theory from which hypotheses can be specified as pointed out in a review by Bringslimark et al. (2009). Another influencing factor might be that occupational health is a result of an interaction between a number of factors at the workplace such as the physical surroundings, the indoor architectural environment, organizational structures, the culture at the workplace, the social climate, management style, characteristics of the work (e.g., workload and job demands), and employees' psychological states, feeling of empowerment, and level of knowledge (Agervold and Mikkelsen, 2004; Morgeson and Campion, 2003; Sonnentag and Frese, 2003).

In conclusion, more in-depth information about real-life people-plant relationships in office settings and how they are influenced by other workplace characteristics is needed to qualify and direct further research. The present study represents an initial attempt to generate this kind of information.

Based on data from a case-study in an office setting, this article illustrates real-life people-plant relationships and how they are mediated by other workplace characteristics. We adopted the broadest possible understanding of the working environment to avoid leaving out important insights. Nevertheless, the study presented here focused on how the employees subjectively perceived that the present ornamental plants affected their working environment and their well-being. Therefore, we did not measure how ornamental plants directly affected the physical work environment (e.g., air humidity or volatile organic chemicals).

\section{Materials and Methods}

Critical realism (CR) was chosen as the ontological foundation, because it facilitates investigating people-plant relationships as interconnected with the rest of the work environment as a whole (Archer, 2004; Blundel, 2007; Danermark, 2002). Stated shortly, CR is an international and multidisciplinary movement in philosophy closely associated with the work of Roy Bhaskar (Archer, 2004). CR holds the basic assumption that the world is structured, differentiated, stratified, changing, and consists of real objects and events existing independently from human knowledge and concepts (Danermark, 2002). According to $\mathrm{CR}$, research should be occupied with what produces the events and not only with investigating the events themselves. This is done by developing theories. How valid a theory is depends on to which extent it can be used to understand and explain phenomena, connections, and processes. Following the epistemological basic assumption in CR that the choice of research approach depends on the characteristics of the phenomenon under investigation and existing knowledge about it, our starting point was a qualitative and inductive research approach (Miles and Huberman, 1994; Patton, 2002). Epistemologically, the qualitative and the inductive research approach is characterized by naturalistic inquiry, emergent design flexibility, and purposeful sampling starting from open-ended specific observations where categories, concepts, and dimensions emerge as the researcher begins to understand the general patterns that exist in the phenomenon under study (Patton, 2002).

As a methodological foundation, we adopted the case-study design (Eisenhardt, 1989; Yin, 2003) and grounded theory (GT) (Charmaz, 2006; Corbin and Strauss, 2008; Glaser, 1998; Glaser and Strauss, 1967). Both methodologies are closely linked to qualitative and inductive research (Patton, 2002).

\section{Implications of the case-study design}

A nested case-study design was applied to include different levels of analysis, thereby avoiding leaving out possible insights (Neergaard, 2007). Four flexible and interrelated levels of analysis were identified inspired by the work of Hatch (2006) and Bronfenbrenner (1980): 1) the physical level defined as the physical structures of the workplace such as the architecture, interior, and the existing ornamental plants; 2) the organizational level defined as the workplace as an organization, including formal and informal organization of the work, the culture, and values of the workplace and management styles, etc.; 3 ) the social level defined as the interaction between the employees in everyday life and the culture among the employees; and 4) the personal level defined as the employees' personal experiences, attitudes, and behaviors toward ornamental plants both at work and at home.

\section{Implications of grounded theory}

Originally, GT was developed in the 1960s by the sociologists Barney G. Glaser and Anselm L. Strauss. They stressed the idea of developing theories by working inductively with empirically collected data (Glaser and Strauss, 1967). The concept of GT is used in two ways: 1) as a set of methods or guidelines for collecting and analyzing data; and 2) as the end product of using this set of methods (Willig, 2001). Today various versions of GT exist. However, they are based on the same principles (Patton, 2002). Inspired by Charmaz (2006), we used GT as a set of systematic but still flexible guidelines for collection and analyzing data to develop theories grounded in the empirical data. The guidelines incorporated in the research design were simultaneously data collection and analysis, coding of data, use of memos, and constant comparative analysis.

GT distinguishes between substantive and formal theory. We aimed at developing a substantive theory, which comprises explanation and understanding of a phenomenon in a particular area or case, whereas in formal theory, the phenomenon is explained and understood in a broader context by abstract and general concepts and specified links between the concepts (Charmaz, 2006; Glaser, 1998).

\section{Data collection}

Starting from fieldwork, a wide range of data collection methods was applied: 1) observations of physical traces; 2) a semistructured group interview combined with a projection technique; 3) semistructured individual interviews; 4) paper-pencil questionnaires; and 5) a collection of written and statistical material about the workplace. The data collection methods are explained in more detail in the following paragraphs.

1) Observations of physical traces were applied to collect data on the physical level of analysis systematically (Zeisel, 2006). In practice, this included taking pictures of all of the present ornamental plants; recording their species, size, and condition; using annotated diagrams to register the placement of the ornamental plants; and taking field notes to record observations of how the ornamental plants were used.

Based on a need to observe how the organizational history and culture influenced the people-plant relationships, the observations of physical traces were conducted during normal working hours while visiting an employee, who knew the workplace's geography, history, and culture well.

2) A semistructured group interview combined with a projection technique was conducted with key persons in relation to occupational health at the workplace (Kvale, 1996; Patton, 2002). A semistructured interview guide formed the starting point for the interview (Kvale, 1996). The interview guide consisted of an introduction and five themes, which were 1) words and concepts for ornamental plants used by the employees; 2) working environment; 3 ) use of ornamental plants at the workplace; 4) ornamental plants related to human well-being; and 5) ornamental plants at home. To each theme a number of sub-themes and questions was formulated as a backup in case the informants had difficulty talking about the themes. In relation to the second theme, a projection technique was applied as the starting point for talking about the relation between occupational health and the use of ornamental plants (Gauntlett, 2007; Patton, 2002). The participants were asked to build a perfect work environment in Lego $®$ (Lego Systems, Inc., Dk-Billund, Denmark) and Playmobil ${ }^{\circledR} \quad$ (Geobra Brandstätter GmbH \& Co. KG, Zirndorf, Germany) (Fig. 1). The product of this exercise then formed the platform for talking about the working environment.

3) Semistructured individual interviews (Kvale, 1996) with four selected employees were conducted as a means to collect data on the personal level of analysis. Because the participants also talked about the interaction with the other employees during the interview, 


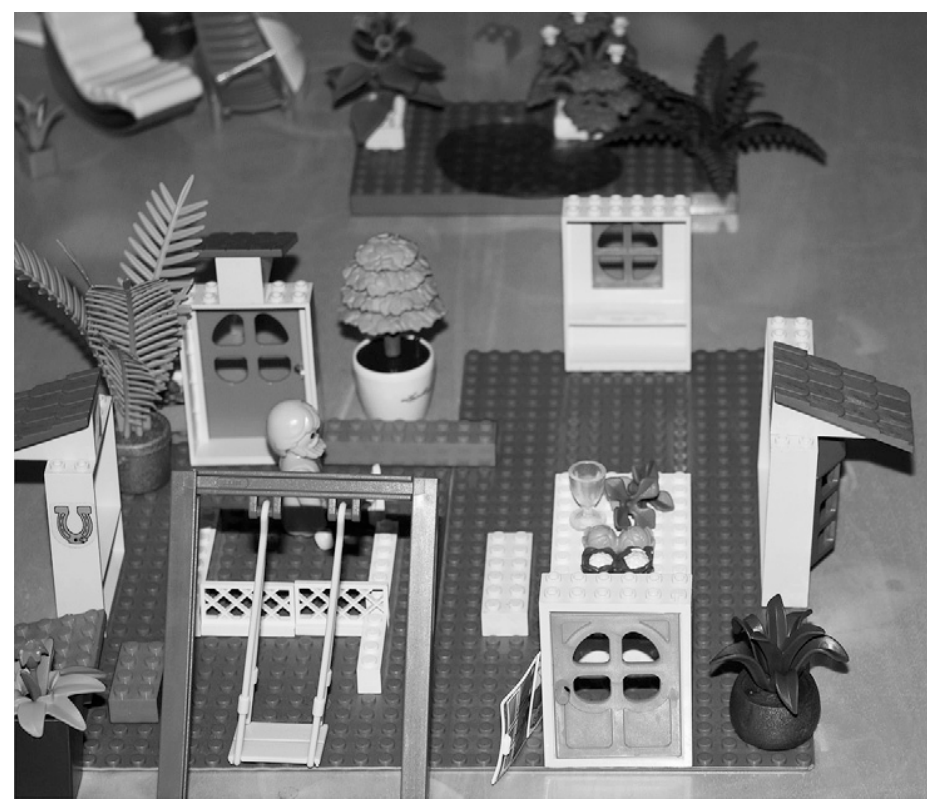

Fig. 1. Example of a product of the projection technique of building the perfect working environment in Lego® and Playmobil®.

it indirectly generated data on the social level of analysis. The individual interviews were conducted with nearly the same interview guide as the group interview; only the projection technique was left out.

4) Collection of written and statistical organizational data about the workplace was applied to generate data on the organizational level of analysis. Especially, we were interested in official data about the organization and the working environment at the workplace such as organization diagrams, mission and goal of the workplace, job descriptions, etc.

5) Paper-pencil questionnaires were administered to all the employees to get an overview of the employees' demographic background and their attitudes toward ornamental plants (Judd et al., 2002). The questionnaire was developed in an inductive manner, although some of the questions were inspired by Bringslimark et al. (2007). The items were mostly closed-ended questions (Judd et al., 2002). Depending on the underlying variable the level of measurement varied, most demographic variables were measured on nominal scales, whereas attitude variables were measured on a 5-point Likert scale (DeVellis, 1991; Fowler, 2008).

\section{Sampling}

The overall sampling strategy was purposive sampling (Neergaard, 2007; Patton, 2002). Because relatively little is known about what constitutes real-life people-plant relationships in office settings, the strategy was to apply broad selection criteria.

The workplace was selected based on two criteria: 1) ornamental plants should at least to some degree be present at the workplace; and 2) the employees should be engaged in office work like secretarial work, handling of information, planning, accounting, etc. (Sundstrom and Sundstrom, 1986).

The participants in the semistructured group interview were those key persons at the workplace who formally or informally took a leading position in relation to occupational health. The participants in the semistructured individual interviews were representative for the population at the workplace.

\section{Sample characteristics}

The selected workplace was a crossdisciplinary unit at a department of a Danish institution taking care of administrative staff functions and secretary tasks to support other staff at the department. Formally, 21 persons plus two at maternity leave were employed at the unit. Informally, three other persons were attached to the unit. All in all, 24 employees were included in the study.

The distribution of gender was seven males and 17 females. On average, the employees were 46 years old spanning from 29 to 66 years. The educational background of the employees varied from no formal training to a PhD level. Most of the employees had been employed at the unit for more than 5 years.

The unit had 19 offices, four meeting rooms, five lunch rooms, and one cantina distributed at two locations. However, the meeting rooms, the lunch rooms, and the cantina were shared with other units. All the employees had their own office or shared an office with one or two colleagues. The unit did not have plants installed and maintained by a professional interior landscaping company.

\section{Material included in the analysis}

All the employees, except one, participated in the study. The leader and two employees participated in the group interview.
Four individual interviews were conducted with four employees: three females and one male varying in age, material status, educational level, and time employed at the workplace. Data included in the analysis were transcripts of all the interviews, notes of observations of physical traces, pictures of the existing ornamental plants and the product of the projection technique, written material about the workplace, and responses from 20 employees on the paper-pencil questionnaire.

\section{Analysis}

The data analysis was started during data collection in which the researcher used memowriting to elaborate on initial insights (Charmaz, 2006). These memos were also used to guide further data collection. After the data collection had finished, an analysis was conducted using different GT coding and memo-writing strategies starting from the semistructured group interview and the semistructured individual interviews. This analysis was performed in three steps using the computer-assisted qualitative data analysis software: QSR Nvivo Version 8 (QSR International, Southport, UK).

Step 1. The first analytic step was initial coding, also called open coding (Charmaz, 2006; Corbin and Strauss, 2008). That is, data were scrutinized using general open-ended questions (e.g., What is this? What is going on? What are people doing?). The result of this first analysis was that small pieces of the data were attached with summarizing labels or names. To stay close to the data, we were careful not using formal concepts when labeling the data. When found reasonable, we used the participants' own wording as labels, also called in vivo coding. In this step, we also used memo-writing to develop the codes.

Step 2. The second step was to start forming core categories. This was initiated by focused coding, which is about investigating under which broad topics the open codes could be categorized (Charmaz, 2006; Lofland, 2006). We did this by creating coding hierarchies by arranging the initial codes in "trees" where the root or the bottom consisted of the core category and the branches consisted of sub-categories (Gibbs, 2007). Memo-writing was used to explore the dimensions and properties of the emerging categories.

Step 3. The third step was axial coding or defining factors constituting the categories and relations among them (Charmaz, 2006). Inspired by Charmaz (2006) and Corbin and Strauss (2008), we did this by looking for 1) conditions that formed the structure of the people-plant relationship by answering questions of where, when, and why; 2) actions and interactions that constituted the people-plant relationship by answering questions of whom and how; and 3) consequences or outcomes of the conditions, actions, and interactions by answering the question of with which consequences. We used memo-writing to sum up the insights gained by axial coding.

To further elaborate on the emerging theory, we also analyzed some of the data in a more quantitative manner by using simple descriptive statistics such as frequencies, 
central tendencies, and dispersion. These data primarily included demographic information about the participants and data about the existing ornamental plants. Inspired by Miles and Huberman (1994), the insights gained were summarized based on development of figures, matrices, and networks.

\section{Results}

All in all, the analysis showed that ornamental plants were an integrated part of the investigated workplace. We found that ornamental plants were present in 23 of the 29 rooms at the workplace. All in all, 106 ornamental plants of different species and sizes were present. Data revealed that the employees regarded ornamental plants as being part of the workplace as shown in Figure 2A. Ornamental plants were not of major concern to the management. The policy was that it was acceptable for the employees to have ornamental plants at the workplace, but employees were responsible for purchasing and maintaining the plants by themselves.

Furthermore, the presence of the ornamental plants was closely linked to the perceived working environment as well as the individual well-being, because ornamental plants were used by the employees in numerous ways with the purpose of either influencing different aspects of the working environment or increasing individual wellbeing (Table 1). However, the use of the ornamental plants was limited and structured by a number of other factors and workplace characteristics as shown in Figure 3. It was found that the ornamental plants were believed to have an impact not only on the perceived working environment and the individual well-being, but also on the competiveness of the workplace as shown in Figure 4. Nevertheless, the actual benefits were the result of interactions among how the ornamental plants were used, characteristics of the ornamental plants (e.g., size, species and condition), and characteristics of the individual employee as shown in Figure 3.

Factors structuring how ornamental plants were used. Seven different factors worked as structuring factors. These factors limited the way in which the ornamental plants could be used and in this sense they constituted a frame in which the people-plant relationships were structured. The structuring factors were 1) culture and traditions; 2) provisional orders; 3) culture and values of the workplace; 4) organizational structures and practices; 5) organizational history; 6) policies about the use of ornamental plants; and 7) characteristics of the architecture. The structuring factors are explained in detail in the following paragraphs.

1) Culture and tradition was a main structuring factor in the present case. The presence of ornamental plants at the workplace was considered to be a widespread and common Danish cultural tradition. As stated by one of the participants: "Aren't there plants at most of workplaces? I think it is normal

A

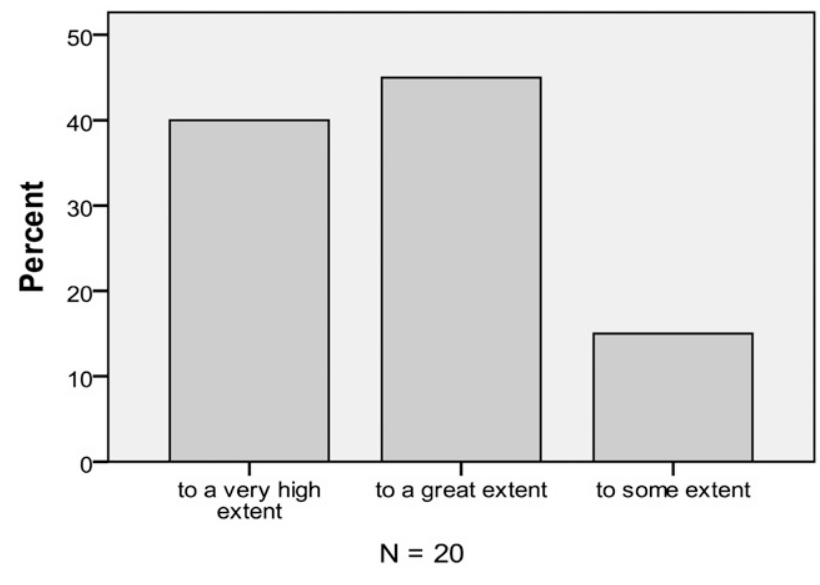

B

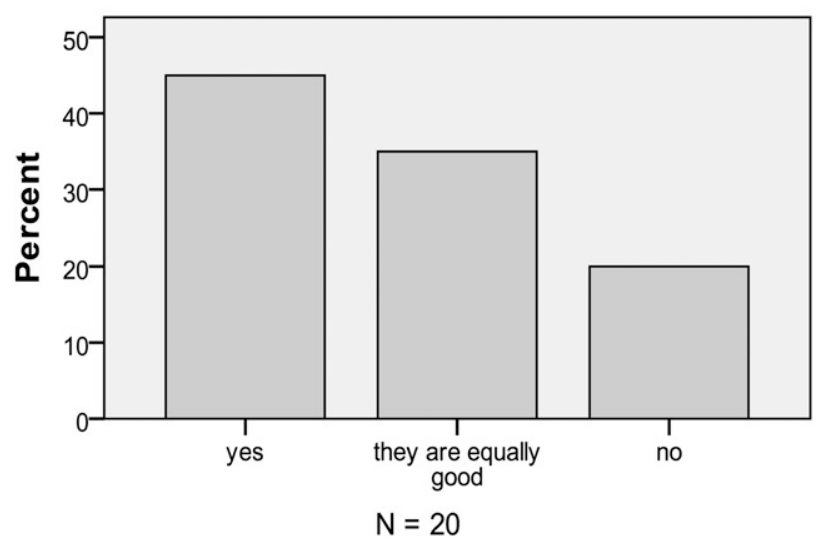

C

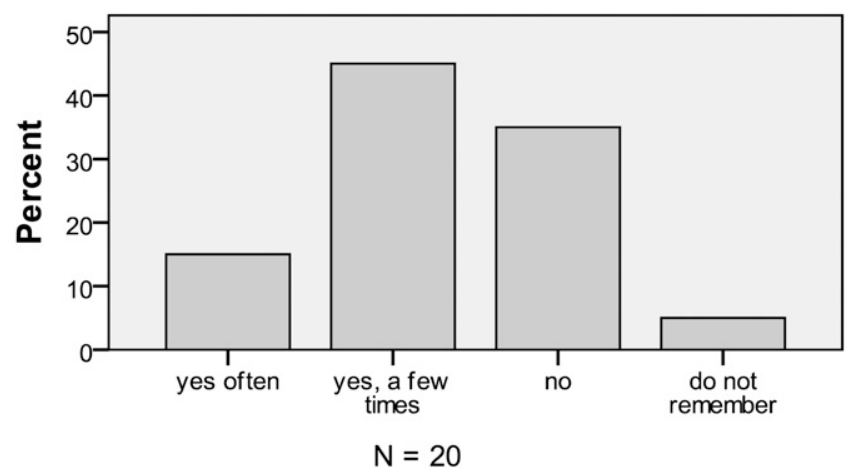

Fig. 2. Examples of data generated by the paper-pencil questionnaire. (A) Answers to the question "To which extent do you think that ornamental plants belong in the workplace?" on a 5-point scale ranging from to a very high extent, a great extent, some extent, a very small extent, and not at all. Nobody answered in the two last categories. Therefore these are not shown. (B) Answers to the question "Do you prefer flowering plants compared with none-flowering plants?" on a categorical scale with the answer possibilities: yes, they are equally good, and no. (C) Answers to the question "Have you brought along ornamental plants to work?" on a categorical scale with the answer possibilities: yes often, yes a few times, no, and do not remember.

to have plants in offices. I don't think I have ever been at an office workplace where plants were not present."

Culture and traditions also influenced the choice of species, size, and placement of the ornamental plants. For example, large plants such as Ficus benjamina standing on the floor were regarded as an integrated part of the interior in an office workplace, especially in open office environments. This was also reflected in the actual choice of species, size, and placement of the present ornamental plants. Twenty-four of the plants were large ornamental plants standing on the floor, mostly of the species Ficus benjamina and different species and cultivars of Dracaena.

Furthermore, culture and traditions played another structuring role, 
Table 1. Overview of ways of using ornamental plants at the workplace.

\begin{tabular}{|c|c|c|c|c|c|}
\hline \multirow{2}{*}{$\begin{array}{l}\text { Involvement }{ }^{\mathrm{z}} \\
\text { Actions }^{\mathrm{y}}\end{array}$} & \multicolumn{2}{|c|}{ Direct } & \multicolumn{3}{|c|}{ Indirect } \\
\hline & Bringing along & Maintaining & Talking about & Looking at & Enjoying \\
\hline & Showing reserves of energy & $\begin{array}{l}\text { Signalling willingness to take } \\
\text { care of thing and make } \\
\text { them grow }\end{array}$ & & $\begin{array}{l}\text { Relaxing in stressed } \\
\text { situations }\end{array}$ & \\
\hline & Improving the indoor climate & $\begin{array}{l}\text { Getting mentally away from } \\
\text { conflict-ridden situations }\end{array}$ & & Coping with boredom & \\
\hline & Keeping mess as at a minimum & Getting exercise & & & \\
\hline & Making people feel welcome & Relaxing in stressful situations & & & \\
\hline
\end{tabular}

'Indicates what characterizes the employees' involvement in the ornamental plants.

'Shows actions constituting the employees' ways of using plants.

${ }^{\mathrm{x}}$ Categorizes intentions behind the certain ways of using plants.

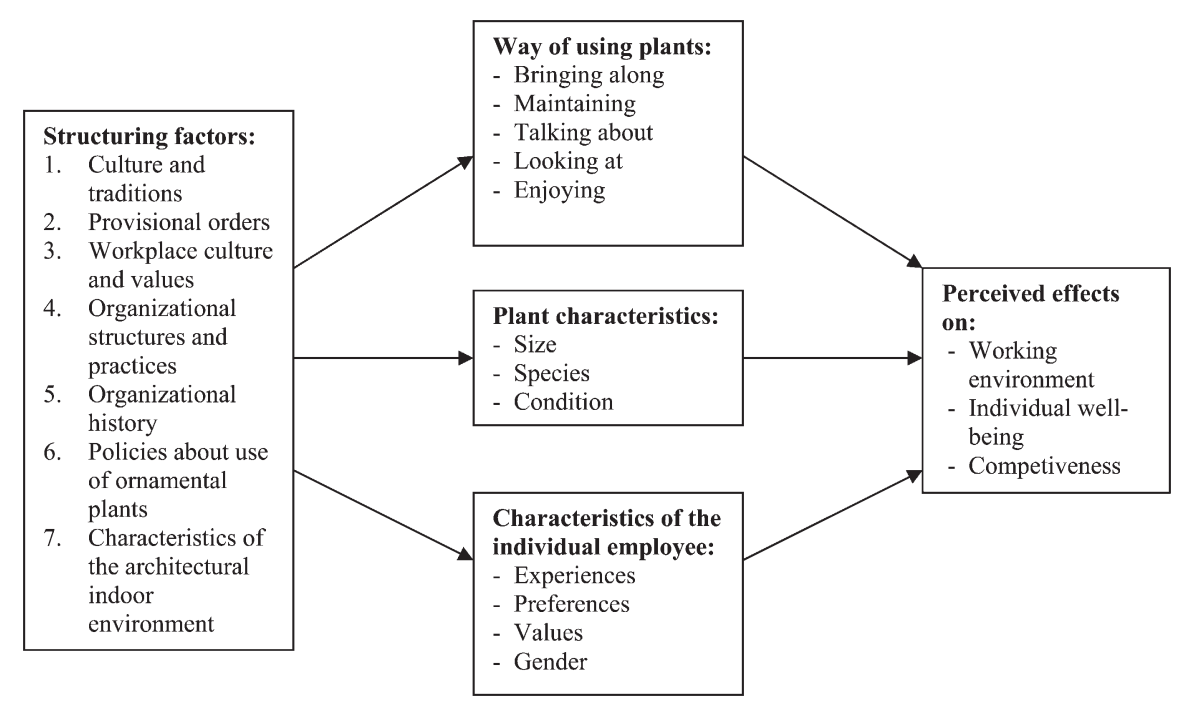

Fig. 3. Model of the relationship between the structuring factors, ways of using plants, plant characteristics, characteristics of the individual employee, and the perceived effect of having ornamental plants at the workplace.

documented by the finding that some species were associated with and used to mark certain cultural events such as Christmas, Easter, or funerals. Some of the employees brought in daffodils for Easter and spruce for Christmas or avoided having certain species (e.g., Calla palustris) as ornamental plants in their offices and homes because they were associated with funerals.

2) Provisional orders existed to regulate the placement of ornamental plants in the present case. The provisional orders regarded safety regulations, which constrained the use of ornamental plants. In consequence, ornamental plants were absent at certain locations, although data indicated that employees would have preferred to have ornamental plants at these locations.

3) Workplace culture and values were important structuring factors by setting up the frame for social acceptable use of ornamental plants. The results showed that ornamental plants were artifacts that were part of the culture at the workplace. Culturally, the ornamental plants gained a special meaning as a result of the common held notion that ornamental plants were living things requiring care. This was evident in a number of ways. First, the employees used expressions like: "it dies," "it grows and develops," "you need to take care of it," and "sometimes you can restore it to life, if it looks dead." Second, the ornamental plants were regarded a specific part of the working environment, which could not be substituted because they were alive. This was illustrated by a unanimous dislike for artificial plants and the general attitude that artificial plants are something negative, because they imitate living plants. Third, the fact that ornamental plants are living things was regarded the reason why ornamental plants contributed positively to the working environment and individual wellbeing. Fourth, the employees reported positive feelings when the plants developed or flowered. This was reflected in the finding that many of the employees preferred flowering ornamental plants as shown in Figure 2B. Fifth, the employees did not change the plants as long as they were just a little alive, even if they looked dead. They were first replaced after several attempts of resuscitation.

Moreover, ornamental plants were often used to express workplace values, as documented by findings showing that some employees volunteered to maintain the plants to show responsibility and one also wanted to take care of the plants to show carefulness toward other employees.

4) Organizational structures and practices were found to be structuring the use of ornamental plants. Especially management style and the nature of the work influenced how the ornamental plants were used.

The way in which the ornamental plants were used was structured by the management style because it provided the frame for using plants without interference from the management. The management style was characterized by freedom with responsibility, meaning that the employees were expected to take care of their tasks but were to a large extent free to decide how to conduct the work as long as they behaved responsibly and solved the tasks they were in charge of. Thus, the employees were free to organize their offices and common facilities as they liked as long as it was done responsibly.

The use of the ornamental plants was structured by the nature of the work, because certain ways of using ornamental plants were regarded as appropriate because the workplace performed services, e.g., some of the employees decorated meeting rooms with flowers for important meetings to make the room comfortable. Also, the fact that the workplace performed services led to concerns about the condition of the ornamental plants and the assumption 


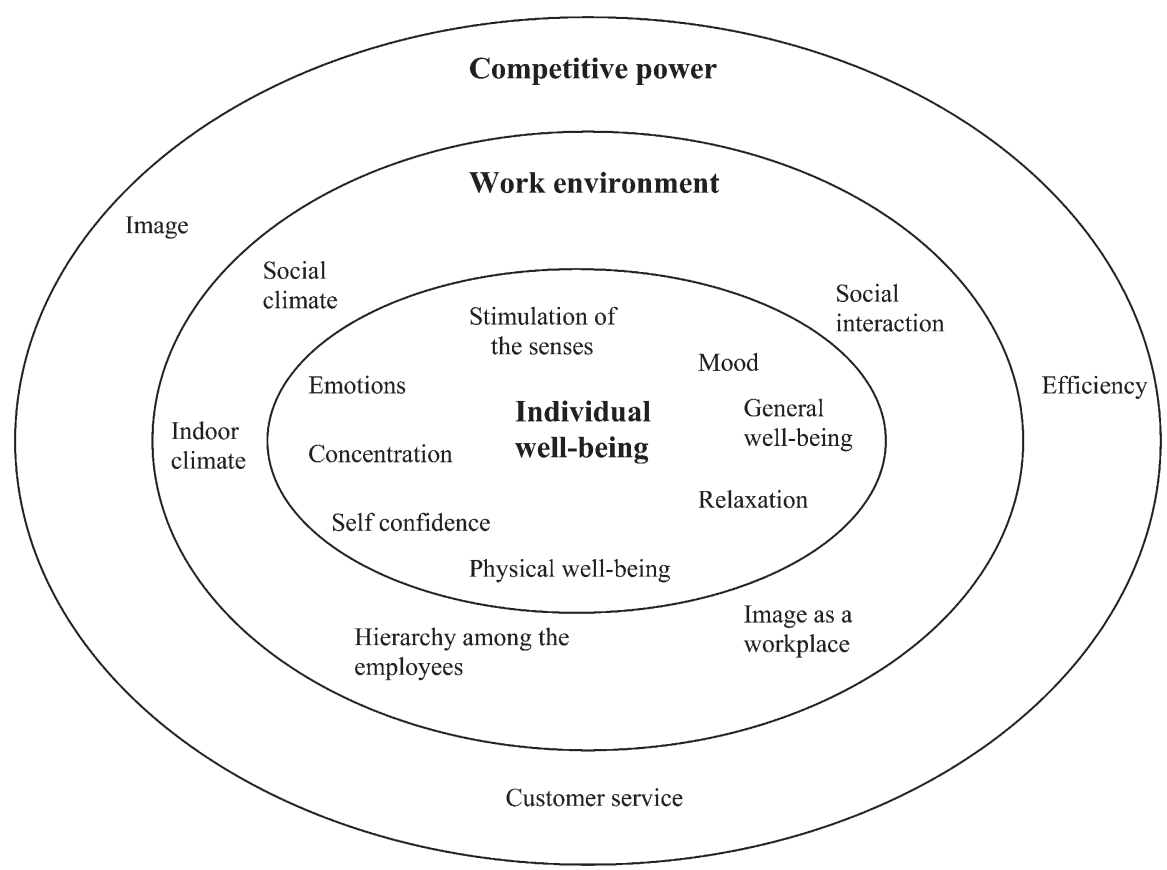

Fig. 4. People-plant relationships affect three levels at the workplace. Plants have an impact on different aspects of competitive power, working environment, and individual well-being.

that neglected plants were harmful in relation to the reputation of being capable of taking care of the tasks responsibly.

5) Organizational history was found to be working as a structuring factor, because the analysis revealed that the people-plant relationships at the workplace were structured by the decisions taken by a former management team. The former management had decided to purchase large ornamental plants and made it part of one of the employees' job descriptions to maintain these plants. The mentioned ornamental plants were still present and it was still part of an employee's job description to maintain them.

6) Policies about use of ornamental plants were structuring the use of ornamental plants in three different ways. First, it allowed direct involvement and active use of ornamental plants, as pinpointed by some of the employees who earlier had worked at workplaces where the employees were not allowed to bring along plants or maintaining them, because a professional interior landscaping company was in attendance to provide and maintain the ornamental plants. Second, the policies constrained which species of ornamental plants were present. Because the employees themselves were responsible for maintenance of the plants, it was important that the applied plant species were able to tolerate periods of neglect like weekends and vacations. It was stressed by the employees that they only brought along species with this feature, which was supported by the finding that all the 41 species present at workplace tolerated long periods of low maintenance. Third, the ornamental plants were personal belongings because the employees were responsible for purchasing the plants as a consequence of the company's policy.

7) Characteristics of the indoor architectural environment such as income of light, space available, layout of the rooms, means for ventilation, and perceived indoor climate were structuring the way the ornamental plants were used. The income of light was stressed as important, because it limited the optimal placement of the ornamental plants and the species that could thrive in the room, whereas space available was highlighted as a factor influencing the appropriate size and number of plants in a room. The layout of the rooms influenced the placement of the plants. Most of the plants were placed in windowsills or on the floor, whereas only a few plants were placed on desks, bookcases, or filing cabinets. Means for ventilation limited the placement and the size of the plants in rooms where the only way of ventilation was opening the windows. Some employees introduced plants in rooms perceived as having a bad indoor climate with the argument that it improved the indoor climate.

Ways of using ornamental plants. The data revealed that generally the employees used ornamental plants in two different ways described by either direct or indirect involvement (Table 1).
Direct involvement was characterized by active and deliberate use of ornamental plants to manipulate different aspects of the surroundings. This included actions as bringing plants into the workplace and maintaining them. Direct involvement was common because $60 \%$ of the employees had brought along ornamental plants as shown in Figure 2C. Many of the employees also maintained the plants and sometimes even maintained plants that were not their own. Motivation for direct involvement was driven by an intention to: 1) manipulate different aspects of the working environment; and 2) express organizational and personal values (Table 1).

We indentified three reasons for bringing ornamental plants into the workplace as a means to manipulate various aspects of the working environment. First, bringing along ornamental plants was considered an important instrument to create a pleasant atmosphere at the workplace. As brought forth by one of the participants: "I believe that they [the ornamental plants] affect you when they are there...in one way or another...it provides a pleasant atmosphere." Moreover, a pleasant atmosphere was considered important to enable effective and healthy work routines. Second, bringing along ornamental plants was considered as a means to improve the physical surroundings. This was reflected by the finding that some of the employees used plants to increase the humidity. Surprisingly, data revealed that some of the employees also brought along ornamental plants to keep the physical surroundings neat and tidy, because they experienced that the ornamental plants took attention away from the piles of paper, thereby making the room look tidy. Third, bringing along ornamental plants was used as an instrument to improve the social working environment, because the presence of ornamental plants was regarded as a way of making people feel welcome and to signal that the employees take care of each other. This was illustrated by some employees giving ornamental plants to colleagues to show a concern about their well-being.

We have discussed the reasons for bringing along plants into the workplace, but also the maintenance of the ornamental plants plays a role. That is, maintaining the ornamental plants was used to induce individual occupational health. In this sense, maintenance of plants was considered an instrument to take a break from the work, get mentally away in conflict-ridden situations, get some exercise, and relax in stressful situations (Table 1).

Furthermore, bringing along as well as maintaining the ornamental plants was used to gain social status, as a result of the finding that bringing and maintaining the plants were associated with high status. Moreover, it was considered as a way of acquiring the role as someone important at the workplace (Table 1).

As an instrument to express organizational and personal values, bringing along plants was done for two reasons. First, 
ornamental plants were brought along to personalize the workplace. This action was mainly driven by an intention of the owner to express him- or herself as a person. Second, ornamental plants were brought along to express organizational and personal values. Some employees brought along ornamental plants to appear as a responsible and caring colleague, whereas others did it to show high standards of services. Additionally, maintaining the ornamental plants was a way of signaling willingness to take care of things in general and make them grow.

Indirect involvement was characterized by more passive and subconscious use of an existing plant. This included actions like talking about the plants, looking at them, or simply enjoying them without being explicitly aware of them (Table 1). We found that most of the employees were indirectly involved by being aware of the presence of the existing ornamental plants at the workplace. Indirect involvement was motivated by a need to socialize and communicate with colleagues, cope with demands in relation to work tasks, and increase general well-being (Table 1).

Talking about the existing ornamental plants was used as a way to socialize and engage in small talk because it was mentioned by one of the participants: "talking about the plants is the same as talking about the weather." Some employees reported that they would talk about the plants to break ice in situations with new people.

The analysis revealed that looking at the existing ornamental plants was an instrument to cope with different demands in relation to the work tasks. Looking at the plants was a way of taking a short mental break from the computer screen, dealing with fatigue or feeling bored, and getting inspiration to solve difficult tasks (Table 1). Furthermore, looking at the plants was done for the same reason as maintaining the plants, namely, to relax in stressed situations (Table 1).

The finding that just being around the ornamental plants increased general wellbeing was linked to the finding that many of the employees enjoyed the plants at a subconscious level as illustrated through this statement expressed by one of the participants: "I am not really aware of them [the ornamental plants]. They are just there in the background spreading a nice atmosphere."

Implications of the people-plant relationships. In the present case, the ornamental plants had an impact on individual well-being, the working environment, and the competiveness of the workplace as shown in Figure 4. In relation to individual wellbeing, it was found that ornamental plants affected mood, general well-being, physical well-being, emotions, concentration, feeling relaxed, self-confidence, and stimulation of senses. It was found that in relation to the working environment, ornamental plants affected the social climate, the image of a workplace, and the interaction and hierarchy among the employees. In relation to the competiveness of the workplace, the analysis showed that ornamental plants were believed to be influencing the general image of the workplace and the efficiency of the employees. This was linked to the finding that ornamental plants were regarded as important means to create a comfortable atmosphere, which again was regarded the basis for conducting effective and high-quality work.

The analysis also revealed that the experienced impact highly depended on the individual employee and the result of interaction among 1) characteristics of the individual employee such as personal experiences, preferences, values and gender; 2) the way the plants were used; and 3) the characteristics of the plants such as size, species, and condition as shown in Figure 3.

Characteristics of the individual employee were found to have an impact on how the ornamental plants were used, which again determined which aspects of the working environment, the individual well-being, or the workplace's competiveness were affected. The characteristics of the individual employee as well as the ornamental plants had an influence on whether the impact was perceived as positive or negative.

Personal experiences of and preferences for certain species were found to be closely linked to the consequence of certain species in relation to emotions and mood. This was documented by the finding that species associated with good times and beloved persons became favorite plants, because they evoked positive feelings and therefore were preferred.

Personal values were found to be guiding the use of ornamental plants, thereby affecting which aspects the individual employee experienced the plants to be influencing.

The data clearly showed that the conditions of the ornamental plants were important. Plants perceived as being in a bad condition generally had a negative effect. Many of the employees reported that being surrounded by ornamental plants in bad conditions invoked negative feelings such as slight irritation. Additionally, these plants were a harmful signal in relation to the image of the workplace as well as the general work environment as illustrated by the statement brought forth in an interview: "Plants in bad condition signal lack of resources and sloppiness." Also, some employees experienced that plants in a bad condition had a slightly negative effect on general well-being. This seems to indicate that the outcome of indirect involvement, as a way of increasing general well-being, is influenced by the perceived condition of the plants.

Another plant characteristic of significance was whether the ornamental plants were flowering. Many of the employees reported that flowering plants in general had a positive impact on the working environment. This was supported by the finding that $45 \%$ of the employees actually preferred flowering plants as shown Figure 2B. Also, many of the employees experienced a great satisfaction when the ornamental plants grew and developed under their care, which again resulted in a higher feeling of self-confidence. However, the analysis also showed that flowering plants sometimes caused negative effects in relation to individual well-being. Some of the employees stressed that several people were allergic to some flowering plants (e.g., lilies). Other employees pointed out that some flowers had a strong smell, which was not acceptable in a working environment.

Although the study was not designed to address the effect of gender, we found that socially acceptable use of ornamental plants was related to gender, illustrated by the participant statement: "Talking about plants is something old people and women do." In addition, some of the male employees avoided using the word "flowers" as synonymous with "ornamental plants," because they considered it too feminine. Instead, they preferred the word "plants." In contrast, many of the female employees preferred the word "flowers" instead of using the words plants or ornamental plants.

\section{Discussion}

The study presented in this article provides information on rich real-life examples of people-plant relationships in a Danish office workplace. In this way, it offers new insights on the people-plant relationships and how they benefit (Thomsen and Müller, 2010). Four main insights were found.

First, the ornamental plants are an integrated part of the workplace and applied in numerous ways driven by intentions to 1 ) manipulate different aspects of the working environment; 2) express organizational and personal values; 3 ) socialize and communicate with colleagues; 4) cope with demands in relation to work tasks; and 5) increase general well-being. In this sense, ornamental plants are not only a simple part of the interior, but are considered to be an instrument used actively and deliberately on the one hand and passively and subconsciously on the other. This indicates that people-plant relationships in office settings cover more that just having ornamental plants and occasionally look at them, which is the use that has been investigated by the existing research (Adachi et al., 2000; Bringslimark et al., 2007; Campbell, 1979; Chang and Chen, 2005; Dravigne et al., 2008; Fjeld, 2000; Fjeld et al., 1998; Larsen et al., 1998; Lohr et al., 1996; Lohr and Pearson-Mims, 2000; Shibata and Suzuki, 2001, 2002, 2004; Shoemaker et al., 1992).

Second, the results of the study reveal that to a large extent the people-plant relationships at the workplace are a social phenomenon, influencing the social climate, hierarchy, and interaction among the employees. In this way, people-plant relationships are not only concerning the employee at a personal level, but also encompass the social level. The documentation of the social aspect of people-plant relationships in offices settings is new, although similar results have been found in a study investigating the impact of indoor gardening on elderly residents of an assisted living facility (Collins and O'Callaghan, 
2007) and psychiatric hospitals (Talbott et al., 1976).

Third, the study documents that use of ornamental plants was structured by a number of factors and workplace characteristics. Especially, company policies regarding the use of ornamental plants seem to play an important role enabling certain ways of applying ornamental plants and excluding others. This implies that people-plant relationships might be cultural and workplace-specific. Therefore, it would be interesting to investigate the role of these factors further.

Fourth, implications of the people-plant relationships were the result of the interplay among the way in which the ornamental plants were used, the characteristics of ornamental plants, and the characteristics of the individual employee. Particularly, the condition of the plants, the annotations connected to specific species, and the personal preferences were important. To a large extent, these factors determined the perceived strength of the effect and whether it was perceived as positive or negative in relation to the working environment, individual well-being, and the competiveness of the workplace. In conclusion, people-plant relationships in the investigated workplace are characterized by being complex, multifarious, and dynamic. Other studies have implied the complexity of people-plant relationship in office settings (Bringslimark et al., 2007; Lohr et al., 1996; Shibata and Suzuki, 2001, 2002, 2004). However, the results of this study might contribute to a more comprehensive understanding of this complexity by offering new insights into its constraints and determinants.

Moreover, the results of the present case confirm the existing research in a number of ways. In relation to productivity, the study showed that the presence of ornamental plants was thought to be associated with efficiency. In addition, some of the employees reported that they looked at the ornamental plants to get inspiration to solve difficult tasks supporting the assumption that the presence of ornamental plants might improve creative task performance. Furthermore, some employees reported that the ornamental plants sometimes acted as a distracter, thereby leading to decreased productivity. These findings confirm findings of the studies by Bringslimark et al. (2007), Larsen et al. (1998), Lohr et al. (1996), and Shibata and Suzuki (2001, 2002, 2004). However, in contrast to findings of some of the existing studies (Shibata and Suzuki, 2001, 2002, 2004), a decrease in productivity seems not to be caused by the task type. Instead, the perceived condition of the plants seems to play a role.

In relation to stress and discomfort symptoms, the results of the present study confirm the assumption that the use of ornamental plants is related to stress reduction because they enhance coping strategies (Bringslimark et al., 2007). In this way, the present study documents certain ways of applying plants (e.g., looking at or maintaining the ornamental plants) served as part of a coping strategy when feeling stressed.
The results also support studies finding that ornamental plants have the ability to improve mood and invoke positive feelings (Adachi et al., 2000; Shibata and Suzuki, 2002, 2004). The results support that the impact is influenced by gender and whether the plants are flowering. However, the present study also shows that species and condition of the plants and the employees' individual preferences were of importance.

The present study describes and analyzes an example of people-plant relationships in a real-life office setting and the outcome of the analysis provides a basis for further empirical and theoretical work. Although the study provides many insights and contributes to a better understanding of the complexity of people-plant relationships in office settings, the study has some limitations. In this context, an important question is to which extent the results can be generalized. It can be assumed that to some extent, similar results can be obtained in other workplaces. However, the study also indicates that people-plants relationships might be culture and workplace-specific and to a large extent are dependent on company policies regarding use of plants. Therefore, a comparative case study focusing on these aspects could qualify the study further. Moreover, the nature of qualitative data makes it difficult to discuss statements regarding causal relations. A research issue that we have not touched is how plants are used to compensate for windowlessness in offices (Biner et al., 1993; Orians and Heerwagen, 1986). Although this is an important issue, we have no data on the topic because all participants in our study enjoyed window views. It would be relevant to further explore this relation in coming studies.

\section{Literature Cited}

Adachi, M., C.L.E. Rohde, and A.D. Kendle. 2000 Effects of floral and foliage displays on human emotions. HortTechnology 10:59-63.

Agervold, M. and E.G. Mikkelsen. 2004. Relationships between bullying, psychosocial work environment and individual stress reactions. Work Stress 18:336-351.

Archer, M. 2004. Critical realism: Essential readings. Routledge, London, UK.

Biner, P.M., D.L. Butler, T.E. Lovegrove, and R.L. Burns. 1993. Windowlessness in the workplace: A reexamination of the compensation hypothesis. Environ. Behav. 25:205-227.

Blundel, R. 2007. Critical realism: A suitable vehicle for entrepreneurship research, p. 4974. In: Neergaard, H. and J.P. Ulhøi (eds.). Handbook of qualitative research methods in entrepreneurship. Edward Elgar, Cheltenham, UK.

Bringslimark, T., T. Hartig, and G.G. Patil. 2007. Psychological benefits of indoor plants in workplaces: Putting experimental results into context. HortScience 42:581-587.

Bringslimark, T., T. Hartig, and G.G. Patil. 2009. The psychological benefits of indoor plants: A critical review of the experimental literature. J. Environ. Psychol. 29:422-433.

Bronfenbrenner, U. 1980. The ecology of human development. Harvard Univ. Press, Cambridge, MA.
Campbell, D.E. 1979. Interior office design and visitor response. J. Appl. Psychol. 64:648-653.

Chang, C.Y. and P.K. Chen. 2005. Human response to window views and indoor plants in the workplace. HortScience 40:1354-1359.

Charmaz, K. 2006. Constructing grounded theory: A practical guide through qualitative analysis. Sage Publ., London, UK.

Collins, C.C. and A.M. O'Callaghan. 2007. Healing gardens for assisted living: An interdisciplinary approach to health education. HortTechnology 18:611-618.

Conklin, E.L. 1978. Interior landscaping. J. Arboricult. 4:73-79.

Corbin, J. and A. Strauss. 2008. Basics of qualitative research: Techniques and procedures for developing grounded theory. 3th Ed. Sage Publ., Thousand Oaks, CA.

Danermark, B. 2002. Explaining society: Critical realism in the social sciences. Routledge, London, UK.

DeVellis, R.F. 1991. Scale development: Theory and applications. Sage Publ., Newbury Park, CA.

Dravigne, A., T.M. Waliczek, R.D. Lineberger, and J.M. Zajicek. 2008. The effect of live plants and window views of green spaces on employee perceptions of job satisfaction. HortScience 43:183-187.

Eisenhardt, K.M. 1989. Building theories from case-study research. Acad. Manage. Rev. 14: 532-550.

Fjeld, T. 2000. The effect of interior planting on health and discomfort among workers and school children. HortTechnology 10:46-52.

Fjeld, T., B. Veiersted, L. Sandvik, G. Riise, and F. Levy. 1998. The effect of indoor foliage plants on health and discomfort symptoms among office workers. Indoor Built Environ. 7:204-209.

Fowler, F.J. 2008. Survey research methods. 4th Ed. Sage Publ., Thousand Oaks, CA.

Gauntlett, D. 2007. Creative explorations: New approaches to identities and audiences. Routledge, London, UK.

Gibbs, G.R. 2007. Analyzing qualitative data. Sage Publ., Los Angeles, CA.

Glaser, B.G. 1998. Doing grounded theory: Issues and discussions. Sociol. Press, Mill Valley, CA.

Glaser, B.G. and A.L. Strauss. 1967. The discovery of grounded theory: Strategies for qualitative research. Aldine Publ., New York, NY.

Hatch, M.J. 2006. Organization theory: Modern, symbolic, and postmodern perspectives. 2nd Ed. Oxford Univ. Press, New York, NY.

Judd, C.M., R.H. Hoyle, and M.J. Harris. 2002. Research methods in social relations. 7th Ed. Wadsworth, South Melbourne, Australia.

Kvale, S. 1996. Interviews: An introduction to qualitative research interviewing. Sage Publ., Thousand Oaks, CA.

Larsen, L., J. Adams, B. Deal, B.S. Kweon, and E. Tyler. 1998. Plants in the workplace-The effects of plant density on productivity, attitudes, and perceptions. Environ. Behav. 30:261281.

Lofland, J. 2006. Analyzing social settings: A guide to qualitative observation and analysis. 4th Ed. Wadsworth/Thomson, Belmont, CA.

Lohr, V.I. and C.H. Pearson-Mims. 2000. Physical discomfort may be reduced in the presence of interior plants. HortTechnology 10:53-58.

Lohr, V.I., C.H. Pearson-Mims, and G.K. Goodwin. 1996. Interior plants may improve worker productivity and reduce stress in a windowless environment. J. Environ. Hort. 14:97-100.

Miles, M.B. and A.M. Huberman. 1994. Qualitative data analysis: An expanded sourcebook. 2nd Ed. Sage Publ., Thousand Oaks, CA. 
Morgeson, F.P. and M.A. Campion. 2003. Work design, p. 423-452. In: Weiner, I.B. (ed.). Handbook of psychology. Vol. 12. Wiley, Hokoben, NJ.

Neergaard, H. 2007. Sampling in entrepreneurial settings, p. 253-274. In: Neergaard, H. and J.P. Ulhøi (eds.). Handbook of qualitative research methods in entrepreneurship. Edward Elgar, Cheltenham, UK.

Orians, G.H. and J.H. Heerwagen. 1986. Adaptations to windowlessness: A study of the use of visual decor in windowed and windowless offices. Environ. Behav. 18:623-639.

Patton, M.Q. 2002. Qualitative research \& evaluation methods. 3th Ed. Sage, Thousand Oaks, CA.

Pearson-Mims, C.H. and V.I. Lohr. 2000. Reported impacts of interior plantscaping in office environments in the United States. HortTechnology 10:82-86.
Shibata, S. and N. Suzuki. 2001. Effects of indoor foliage plants on subjects' recovery from mental fatigue. N. Amer. J. Psychol. 3:385-396.

Shibata, S. and N. Suzuki. 2002. Effects of the foliage plant on task performance and mood. J. Environ. Psychol. 22:265-272.

Shibata, S. and N. Suzuki. 2004. Effects of an indoor plant on creative task performance and mood. Scand. J. Psychol. 45:373-381.

Shoemaker, C.A., K. Randall, P.D. Relf, and E.S. Geller. 1992. Relationships between plants, behavior, and attitudes in an office environment. HortTechnology 2:205-206.

Sonnentag, S. and M. Frese. 2003. Stress in organizations, p. 453-492. In: Weiner, I.B. (ed.). Handbook of psychology. Vol. 12. Wiley, Hokoben, NJ.

Sundstrom, E. and M.G. Sundstrom. 1986. Work places: The psychology of the physical envi- ronment in offices and factories. Cambridge Univ. Press, New York, NY.

Talbott, J.A., D. Stern, J. Ross, and C. Gillen. 1976. Flowering plants as a therapeutic environmental agent in a psychiatric hospital. HortScience 11:365-366.

Thomsen, J.D. and R. Müller. 2010. Plants for a better life-People-plant relationships in indoor work environments. Acta Hort. 881:837841.

Willig, C. 2001. Introducing qualitative research in psychology: Adventures in theory and method. Open Univ. Press, Buckingham, UK.

Yin, R.K. 2003. Case study research: Design and methods. 3th Ed. Sage Publ., Thousand Oaks, CA.

Zeisel, J. 2006. Inquiry by design: Environment, behavior, neuroscience in architecture, interiors, landscape, and planning. W.W. Norton \& Company, New York, NY. 